\title{
Nutritional functionality of foods
}

\author{
BY PAULUS M. VERSCHUREN \\ Unilever Research Laboratory, Vlaardingen, The Netherlands
}

The relationships between diet and health are becoming more and more substantial, in particular with respect to the most prominent chronic diseases in developed countries, i.e. cardiovascular disease and cancer. As a consequence health authorities advise populations on ways to improve their lifestyle including dietary habits. However, despite consumer interest in health-related issues changing nutrition habits remains difficult. Nevertheless, consumers expect food products to be healthy, as well as tasty and functional. This has triggered the food industry to look for opportunities to improve existing products as well as for developing new food products which can contribute to a healthy balanced diet. The basis for such developments is provided by the outcomes of epidemiological studies, e.g. fruits and vegetables protect against cancer and cardiovascular disease. Currently, a myriad of in vivo and in vitro investigations with many potentially active components of fruits and vegetables are being carried out to unravel their nutritional functionality. These studies focus on the underlying mechanisms of the potential health benefits, including their bioavailability and antioxidant properties. Through close collaboration of both industry and academia it may become increasingly feasible to link food ingredients to specific health benefits. Such a development would allow the food industry to provide the consumer with food products tailored to their functional, nutritional and health needs.

In past decades the link between lifestyle and health has become increasingly evident. Today, diet alone is suggested to account for almost one-third of cancer deaths in Western populations, and along with tobacco, is amongst the most modifiable environmental factors which affect human health (Greenwald, 1996; Trichopoulos et al. 1996; Willett et al. 1996). Less strong, but similar, evidence exists for cardiovascular disease (Knekt et al. 1994; Gillman et al. 1995; Kushi et al. 1995). Dietary habits are thus regarded as an important element of one's lifestyle. The reason that populations in industrialized countries are increasingly facing major diet-related health problems, of which cardiovascular disease and cancer are most prominent, partly lies in the fact that over time dietary habits have changed significantly. Consumer demands have evolved from the need for foods to simply survive, to the intake of a variety of food products for enjoyment and indulgence. Results from nutrition sciences have triggered a growing awareness that diet-related health problems can be modified through dietary change. As a consequence, altered dietary habits are being propagated worldwide. Despite the fact that most consumers in Western countries are more or less aware of the importance of changing to a healthier diet by lowering their energy intake, eating more plant foods like fruits and vegetables, less fat and saturated fat, and less table salt, remarkably few are actually able to adhere to these recommendations. In order to maintain health and to combat diseases of affluence the consumer is now looking for food products which have less adverse effects on health and which can positively enhance health and well-being. In this respect it is important to know what aspects actually drive consumer choices and what contributions are made by nutrition scientists.

\section{CONSUMER ASPECTS}

From a consumer point of view, apart from costs, there are other important elements which affect their choice of food products, i.e. taste, functionality and health. Although foods are 
recognized by texture, form and colour, they are mostly appreciated by taste. Taste is $95 \%$ flavour perception, and an estimated $1 \%$ of all genes in human subjects is devoted to the perception of flavour, indicating its importance in another way. For the consumer the aspect of taste is simple to judge and the consumer will only purchase foods which have a good taste. If the taste is not right it will not be bought. Thus, flavours significantly affect the acceptance of foods and, therefore, are essential elements in food products. More and more is known about flavour components and combinations in foods and it is a challenge for the food industry to get the taste right. The same can be said for functionality and convenience; for example, a margarine should be spreadable straight from the fridge, the viscosity of sauces should be just right, and differences in family size require variable packaging and portionable sizes.

However, judging the health aspects of foods is less easy for the consumer. Whether a particular food product fits into a healthy diet is something which needs to be communicated by the food producer. Because of consumer demands for foods which positively contribute to health, a major responsibility lies with the food industry. They should not only develop foods which actually help consumers to compose a healthy balanced diet, but also ensure that the communication about products is scientifically sound and easy to understand. Consumer sciences indicate that throughout Europe consumer views on health issues are not identical. In the UK and Germany, for example, the majority of consumers are particularly concerned about heart disease, whilst in France, stress is a key worry. This implies that food industries in these countries also need to tailor their development and marketing to these local differences.

\section{SCIENTIFIC ASPECTS}

When considering improving food products, it is important to know their composition, nutritional quality and position in the diet, as well as consumer use. The evaluation of these aspects may lead to options for the food industry for product improvement. If through research a particular product innovation is expected to have health-promoting properties, this is where bioavailability and efficacy of the product or product ingredient comes in. Reviewing options for improvement requires initial information on health effects of foods or food ingredients through epidemiological studies. Knowledge needs to be built up on the suggested relationships between a food or a food ingredient and their function in the human body. This work could involve a range of studies including in vitro and in vivo research both in animals and human subjects. As is it not always practical and/or ethical to carry out studies in human subjects to evaluate the relationship between foods and particular diseases much effort is put into identifying appropriate biomarkers for disease and well-being.

For example, epidemiological studies show that a high intake of fruits and vegetables is associated with a reduced risk of cancer and heart disease. Block et al. (1992) suggest in a meta-analysis that the risk of death by many non-hormone-dependent cancers can be reduced approximately twofold in subjects who consume relatively high amounts of fruits and vegetables. Fruits and vegetables are rich in carotenoids which have antioxidant properties. Data from experimental studies demonstrated a relationship between antioxidant activity, free-radical scavenging, oxidative damage, changes in cell function and disease endpoints such as cancer and heart disease (Greenwald, 1996). In order to test the hypothesis that diets rich in fruits and vegetables reduce the risk for cancer and heart disease, life-long intervention studies would be required. Testing for the effects of foods or food ingredients on disease pathogenesis would be too expensive, impractical and unethical. Consequently, much attention is spent on the development of biomarkers for 
disease. For example, in colo-rectal carcinogenesis, a cancer which ranks second as a cause of death in the United States, several potential biomarkers have been identified. These vary from single base mutations, to particular macroscopic features of colon epithelium, such as aberrant crypts. Aberrant crypt multiplicity is now considered a reliable marker for colon cancer in carcinogen-induced rat models (Wingo et al. 1995). As oxidative damage may play an important role in the development of this disease the effects of antioxidants on this biomarker are presently under study. If adequate proof is obtained that particular food ingredients, i.e. antioxidants, have an impact on health it is important to know the bioavailability and efficacy of these ingredients. This will then allow the food industry to increase the antioxidant content of plants through plant breeding, to optimize antioxidant retention during processing, to enhance the release of antioxidants from food products or to fortify food products with antioxidants.

Food products which provide a positive contribution to health beyond traditional nutritional values are regarded as functional foods. The concept of functional foods has been raising much discussion within the member states of the EU. Up to now, no common position has been achieved concerning either the scientific status or the regulatory position within the EU. Despite the many definitions of functional foods which are presently in place, the general feeling of the scientific community is that functional foods should stay foods and that through modulating specific physiological functions they should have a proven health-promoting effect allowing the use of claims. In this respect, it is important to note that communication to the consumer about functional foods should always be scientifically sound, understandable for the target population, and consistent over the world.

\section{REFERENCES}

Block, G., Patterson, B. \& Subar, A. (1992). Fruit, vegetables and cancer prevention: a review of the epidemiological evidence. Nutrition and Cancer 18, 1-29.

Gillman, M. W., Cupples, L. A., Cagnon, D., Millen Posner, B., Ellison, R. C., Castelli, W. P. \& Wolf, P. A. (1995). Protective effect of fruits and vegetables on development of stroke in men. Journal of the American Medical Association 273, 1113-1117.

Greenwald, P. (1996). Chemoprevention of cancer. Scientific American September issue, 64-67.

Knekt, P., Reunanen, A., Jarvinen, R., Seppanen, K., Heliovaara, M. \& Aromaa, A. (1994). Antioxidant vitamin intake and coronary mortality in a longitudinal population study. American Journal of Epidemiology 139 , 1180-1189.

Kushi, N. I., Lenart, E. B. \& Willett, W. C. (1995), Dietary antioxidant vitamins and death from coronary heart disease in postmenopausal women. American Journal of Clinical Nutrition 61, 1407S-1415S.

Trichopoulos, D., Li, F. P. \& Hunter, D. J. (1996). What causes cancer? Scientific American September issue, 58-63.

Willett, W. C., Colditz, G. A. \& Mueller, N. E. (1996). Strategies for minimizing cancer risk. Scientific American September issue, 50-57.

Wingo, P. A., Tong, T. \& Bolden, S. (1995). Cancer statistics 1995. Cancer Journal for Clinicians 45, 8-30. 